\section{A NETWORK PERSPECTIVE OF NECROTISING ENTEROCOLITIS REQUIRING SURGERY}

doi:10.1136/archdischild-2012-302724.1380

M Nash, M Passant, V Rasiah. South West Midlands Neonatal Network, Birmingham, UK

Introduction Necrotising-enterocolitis is the commonest gastrointestinal emergency in very low birth weight (VLBW) infants. The incidence is around 7-per-100 births of VLBW infants. Confirmed cases require aggressive medical therapy, nil-by-mouth for 7-14 days and triple antibiotic therapy. Timely surgical intervention is necessary in those who perforate or are critically ill.

Aim To give a network perspective on the diagnosis, management and outcome of infants affected by NEC focusing on those requiring surgery.

Method Data was retrospectively collected from the Badger database over two years for babies within the South-West Midlands Newborn-Network with a diagnosis of NEC. Babies who were treated for at least 7 days were included, focusing on those requiring surgery.

Results

Abstract 1380 Table 1

\begin{tabular}{ccccc}
\hline & Distinct admissions & $<\mathbf{1 5 0 0 g}$ & Diagnosis NEC & NBM $>$ 7days \\
\hline Number & 8750 & 1407 & 266 & 161 \\
\hline
\end{tabular}

Abstract 1380 Table 2 Outcome

\begin{tabular}{lcccc}
\hline & Number & Survived to discharge & Died & Mortality \\
\hline Surgical & 56 & 48 & 8 & $14 \%$ \\
Conservative & 105 & 88 & 17 & $16 \%$ \\
Overall & 161 & 136 & 25 & $15 \%$ \\
\hline
\end{tabular}

Discussion The standardised use of Badger information system across the network allowed us to audit the outcome of babies with NEC within our network. This is limited by the inter-user variation in quality and detail of information entered.

The National Neonatal Collaborative Necrotizing Enterocolitis Study is currently underway. Our audit provides some insight into the limitations of using Badger alone as a source of information. We need to improve the quality of data entered into Badger.

\section{GASTROINTESTINAL PERFORATIONS IN NEONATAL PERIOD}

doi:10.1136/archdischild-2012-302724.1381

${ }^{1} \mathrm{E}$ Blevrakis, 'C Seremeti, 'N Partalis, ${ }^{1} \mathrm{O}$ Dede, ${ }^{2} \mathrm{~A}$ Alegakis, ${ }^{3} \mathrm{E}$ Korakaki, ${ }^{3} \mathrm{C}$ Gianakopoulou, ${ }^{1} \mathrm{G}$ Sakellaris. 'Department of Paediatric Surgery, University Hospital of Heraklion, Crete; ${ }^{2}$ Department of Toxicology, University of Crete; ${ }^{3}$ Neonatal Intensive Care Unit, University Hospital of Heraklion, Crete, Heraklion, Greece

Aim This report describes our experience concerning gastrointestinal perforation (GI) due to Necrotizing enterocolitis (NEC) during a 10 year period.

Method The cases of 27 GI perforations, which were treated in our hospital, were retrospectively reviewed.

Results All patients were neonates and infants up to the age of 2 months. The study population consisted of 16 boys (59.3\%) and 11 girls $(40.7 \%)$. Twenty one $(77.8 \%)$ neonates were pre-term and the median gestational age was 28 weeks. Twenty four (88.9\%) cases of perforation underwent laparotomy. The overall mortality was $63 \%$. Seventy six percent of the pre-term neonates and only $16.7 \%$ of the full-term died.

Conclusions GI perforation is still connected with a high mortality rate, with NEC being the main cause of death. The neonates who did not undergo surgery all died.

\section{INTRODUCING ROUTINE PROBIOTICS FOR PRETERM NEONATES - NEPEAN HOSPITAL SYDNEY AUSTRALIA APPROACH}

doi:10.1136/archdischild-2012-302724.1382

1,2G Deshpande, 1,2V Shingde, 1,2L Downe, 2,3M Leroi, ' J Xiao. 'Neonatology, Nepean Hospital Sydney; ${ }^{2}$ Nepean Clinical School, University of Sydney, Kingswood, NSW, Australia; ${ }^{3}$ Microbiology, Nepean Hospital Sydney, Kingswood, Austria

Background Probiotic supplementation significantly reduces the risk of mortality and $\geq$ Stage II necrotising enterocolitis in preterm neonates. (Deshpande et al Lancet 2007, Pediatrics 2010). Based on the current evidence, experts favour routine probiotic supplementation in preterm neonates, if safe and effective products are available. We implemented a stepwise plan to introduce probiotics in our regional tertiary neonatal centre.

Step wise approach:

1. Comprehensive literature review to develop evidence based guidelines for optimal use of probiotics in preterm neonates.

2. Identifying a suitable product based on the systematic review of RCTs in preterm neonates, and probiotics listed by Therapeutic Goods Administration, (TGA).

3. Approval of selected probiotic product (Infloran) from local area drug and therapeutics committee.

4. Endorsement from local Ethics Committee.

5. Authorised Prescriber application to TGA via Pharmacy Department.

6. Approval from authorities regarding importing probiotic product from overseas.

7. Independent quality assessment (Taxonomy confirmation, antibiotic susceptibility, and osmolarity).

8. Further assessment for ruling out contamination from a TGA approved laboratory.

Results 2/19 locally available products were potentially appropriate for use in preterm neonates but turned out suboptimal in quality despite manufacturer's data. (Patole et al PSANZ 2010) An overseas proven probiotic product was selected (Infloran) and imported after following above stepwise approach. After vigorous quality assessment Infloran was introduced routinely in preterm neonates at Nepean Hospital Sydney.

Conclusion Safe and effective probiotic product is accessible in Australia for routine use in preterm neonates. Further co-operation is needed between industry, regulatory authorities and clinicians to simplify this process.

\section{IMPROVEMENT OF GUT MICROBIOTA AFTER PROBIOTIC TREATMENT OF PREMATURE NEONATES FOR THE PREVENTING OF NECROTIZING ENTEROCOLITIS}

doi:10.1136/archdischild-2012-302724.1383

\section{B Smith. Statens Serum Institut, Copenhagen, Denmark}

Purpose Necrotizing enterocolitis (NEC) is the most common acquired disease of the gastrointestinal tract in premature neonates. Probiotic supplementation with Lactobacillus rhamnosus and Bifidobacterium lactis was introduced to reduce NEC and potentially provide benefits for the preterm neonates. We study if premature neonates were colonized with $L$. rhamnosus and B. lactis and investigated what influence probiotics had on clinical outcomes.

Methods Faecal samples and clinical information from neonates before and after the introduction of probiotic were collected. 254 premature neonates without probiotics and 101 premature neonates with probiotics all less than 30 weeks of gestation were enrolled. Fecal samples and clinical information during the first month of life from all premature neonates were collected.

Results Clinical information on all premature neonate regarding, gestational age, birth weight, probiotic, antibiotics, food, NEC and dead were collected. A total of 461 feacal samples from premature 